\title{
Las Ocupaciones Humanas del Sitio Arqueológico de Santa Inés, Laguna de Tagua Tagua, Chile Central
}

\author{
Human occupation of the Santa Inés site, Tagua Tagua basin, \\ central Chile
}

Donald Jackson ${ }^{1}$, Eugenio Aspillaga ${ }^{1}$, Xose-Pedro Rodríguez ${ }^{\text {II }}$, Douglas JaCKson ${ }^{\mathrm{III}}$, Francisca Santana ${ }^{\mathrm{IV}}$ y CÉSAR MÉNDEZ

\section{RESUMEN}

El estudio de las evidencias arqueológicas y bioantropológicas del sitio arqueológico de Santa Inés, situado en la ex laguna de Tagua Tagua (Chile Central), y sus nuevas edades radiocarbónicas, han permitido definir este sitio como un asentamiento habitacional con varios eventos ocupacionales asociados a prácticas funerarias. Las primeras ocupaciones corresponderian a cazadores-recolectores del Holoceno Medio y Tardio y el último a un evento del Alfarero Temprano. Poseen fuertes afinidades con el cercano asentamiento de Cuchipuy. Como conjunto constituyeron parte de un patrón de asentamiento en torno a los recursos lacustres de la cuenca de Tagua Tagua.

Palabras claves: Patrón de Asentamiento, Adaptaciones Circunlacustres, Chile Central.

\section{Abstract}

The study of archaeological and bioanthropological evidence from the Santa Inés site (central Chile), together with new radiocarbon dates available, have allowed us to define this site as a residential settlement with several occupational events associated with funerary practices. The first occupations correspond to mid-to-late Holocene hunter-gatherers, while the last event to the Early Ceramic Period. Santa Inés presents strong affinities to the Cuchipuy site located in the vicinity. As a whole, they formed part of a settlement pattern clustered along the shoreline of the Tagua Tagua basin and its lake-basin resources.

Key words: Settlement Pattern, Lake-basin Adaptations, Central Chile.

i Departamento de Antropología, Facultad de Ciencias Sociales, Universidad de Chile. Correo.e: djackson@ uchile.cl; easpilla@uchile.cl; cmendezm@uchile.cl.

ii Área de Prehistoria, Departamento d'Historia i Geografía, Universitat Rovira i Virgili, España. Correo-e: josepedro.rodriguez@urv.cat.

iii Sociedad Malacológica de Chile: Correo-e: sillutus@hotmail.com.

iv Research Laboratory for Archaeology and the History of Art, University of Oxford. Correo-e: francisca. santanasagredo@st-hughs.ox.ac.uk 


\section{INTRODUCCIÓN}

La cuenca de Tagua Tagua en Chile Central $\left(-34^{\circ} \mathrm{S}\right)$ albergó una extensa laguna, generando un polo de atracción para las ocupaciones humanas desde el Pleistoceno Final al Holoceno Tardío. Este ambiente con abundancia y diversidad de recursos fue lugar de campamentos de grupos Paleoindios y articuló asentamientos de cazadores recolectores del Arcaico y posteriormente del período alfarero.

Las primeras ocupaciones en torno a la laguna (hoy drenada) corresponden a grupos Paleoindios identificados en Tagua Tagua 1 (Montané 1968) y Tagua Tagua 2 (Núnez et al.1994), fechados hacia los 13233 12693 y 11803 - 11233 ańos cal AP respectivamente. Se trata de ocupaciones esporádicas orientadas a la caza de fauna actualmente extinta que incluyó, entre otras especies, mastodonte y caballo nativo. Estos megaherbívoros se congregaron en torno a la cuenca, utilizándola como un ecorrefugio ante el advenimiento de condiciones regionales cada vez más áridas (Núnez et al. 1987, Valero-Garcés et al. 2005).

Con posterioridad, la cuenca continúo como foco de atracción para grupos cazadores y recolectores del Holoceno Temprano. Sitios como Cuchipuy muestran una larga secuencia ocupacional asociado a contextos funerarios datados entre los 8870 a 5750 años cal AP. (Kaltwasser et al. 1983). Por otra parte, en los niveles superiores de Tagua Tagua 1 y 2 se presentan ocupaciones de cazadores-recolectores (Montané 1968, Núnez et al. 1994), afines a las identificadas en Cuchipuy y fechadas hacia los 7274 6731 años cal AP (Durán 1980). Estos contextos muestran grupos cazadoresrecolectores económicamente más diversificados con asentamientos de actividades múltiples, fuertemente orientados al aprovechamiento de los recursos lacustres.

Finalmente y con posterioridad a los cazadores-recolectores, la cuenca de Tagua Tagua es ocupada por grupos Alfareros datados entre los 610 años d.C. y los 1300 años d.C. (Cáceres et al. 1995, 2000; Westfall et al. 2001). Se trata de asentamientos habitacionales que atestiguan ocupaciones más estables y que continúan aprovechando, entre otros recursos, los lacustres.

En este marco, el sitio de Santa Inés, localizado en la margen norte de la ex laguna de Tagua Tagua, y excavado intensivamente en la década del '80, proporciona relevante información contextual no estudiada previamente sobre las ocupaciones humanas de los cazadores-recolectores del Holoceno Medio y Tardío, así como de grupos alfareros que ocuparon 
este asentamiento. En este contexto, se estudian las evidencias culturales, se obtienen nuevas edades radiocarbónicas y se plantean algunas relaciones respecto a los patrones de asentamientos en torno a la cuenca de Tagua Tagua, permitiendo plantear que los cazadores-recolectores y posteriormente grupos alfareros de Chile Central, generaron un patrón de asentamiento en torno a sistemas lacustres con recursos estables a lo largo de gran parte del Holoceno.

\section{ÁreA DE ESTUdio y ANTECEDENTES PALEOCLIMÁTICOS}

La actual ex laguna de Tagua Tagua se ubica en la comuna de San Vicente de Tagua Tagua, provincia del Cachapoal, en la región del Libertador Bernardo O’Higgins (Chile Central) (Figura 1).

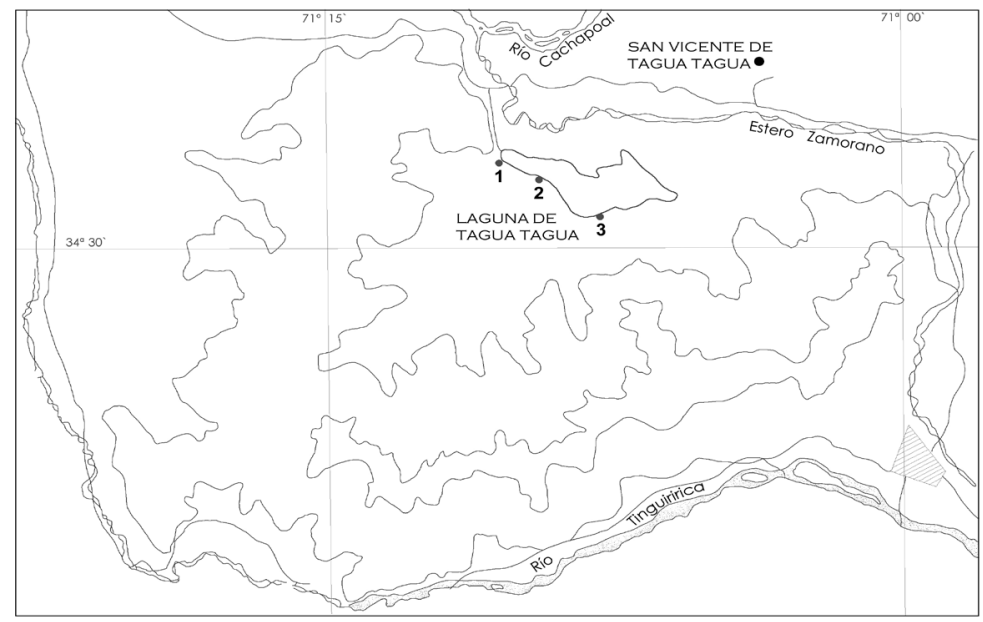

Figura 1: Cuenca de Tagua Tagua y ubicación de sitios mencionados en texto. 1) Tagua Tagua 1 y 2, 2) Santa Inés y 3) Cuchipuy.

Figure 1: Tagua Tagua basin and its relation to the sites mentioned in the text. 1) Tagua Tagua 1 and 2; 2) Santa Inés; 3) Cuchipuy.

El ambiente actual de la región corresponde a un clima templado con lluvias invernales y una estación seca prolongada (Fuenzalida 1965). La vegetación corresponde al piso vegetacional de "bosque esclerófilo mediterráneo andino de Quillaja saponaria y Lithrea caustica" (Luebert y Pliscoff 2006). En los sectores más altos en torno a la cuenca (500 msnm) se observan formaciones boscosas de Nothofagus obliqua y $N$. glauca. La temperatura promedio anual es de $14,5^{\circ} \mathrm{C}$ y las precipitaciones alcanzan un promedio de $578 \mathrm{~mm}$ anuales (Luebert y Pliscoff 2006). Por otra parte, el 
área presenta importantes cursos hidrográficos como el río Cachapoal y el estero Zamorano, así como la antigua cuenca de Tagua Tagua, sobre la cual existen diversas descripciones que atestiguan la diversidad vegetacional y faunística que albergó (Ovalle [1646] 1969, Rosales [1670] 1877, Gay [1831] 1911, Darwin [1909] 2004).

El cordón montańoso que rodea la cuenca de Tagua Tagua se ubica en el margen oriental de la cordillera de la Costa generando una "rinconada" de origen tectónico donde la laguna correspondería a un área deprimida o fosa (Varela 1976a, 1976b). Sus depósitos estratigráficos muestran dos unidades estratigráficas; una inferior de depósitos de ceniza volcánica pumicíticas atribuida al Pleistoceno Medio, y una unidad superior denominada Formación Laguna de Tagua Tagua, constituida por ocho miembros de origen lacustre (Varela 1976a, 1976b).

Varios registros sedimentológicos, geoquímicos y polínicos se extienden durante los últimos 46000 años (Heusser 1983, 1990; ValeroGarcés et al. 2005) y coinciden en que durante el Pleistoceno existieron comunidades vegetales que requirieron de un régimen de precipitaciones mayor al actual (Heusser 1983, 1990; Valero-Garcés et al. 2005). El registro sedimentario caracterizado por arcillas finamente laminadas sugiere el desarrollo de un lago profundo y extenso bajo condiciones de un clima frío y lluvioso (Varela 1976a, 1976b; Valero-Garcés et al. 2005) que albergó importantes contingentes de herbívoros de gran tamaño (Varela 1976b, Núnez et al. 1994). Los resultados de los análisis polínicos indican altos valores en Nothofagus tipo dombeyi y Prumopitys andina para el intervalo entre los -46000 y 21500/18000 ańos cal AP., y sugieren condiciones de humedad semejantes a las de la Región de Los Lagos $\left(-41^{\circ} \mathrm{S}\right)$ (Heusser 1983, 1990; Valero-Garcés et al. 2005). Entre los -21500 y 13790 años cal AP., la variabilidad de las facies sedimentarias sugiere una alta inestabilidad en el tamaño del lago, situación que cambia en el intervalo entre -13790 a 11790 años cal AP., donde los datos apoyan la existencia de un lago profundo (Valero-Garcés et al. 2005). Estas condiciones se aparejan con profundos cambios en la vegetación, primero entre -21500 y 13790 años cal AP. indicando una transición desde condiciones muy frías y húmedas hacia una desecación y, entre los -13790 a 11790 años cal AP., un decrecimiento significativo en los taxa arbóreos, que prácticamente desaparecen hacia el final de la zona. Las condiciones para la transición entre el Pleistoceno y Holoceno son de profundo cambio. A la desaparición de taxa arbórea se suman datos sedimentológicos que apoyan la existencia de un lago muy bajo (Heusser 1983, 1990; Valero Garcés et al. 2005). De hecho, la posición 
estratigráfica del contexto de Tagua Tagua 2 en una discordancia erosiva (entre los Miembros 5 y 6) apoya la idea de una amplia e irregular superficie erosiva producto de la desecación del cuerpo lagunar (Varela 1976b).

En este contexto las evidencias estratigráficas y edades radiocarbónicas aquí presentadas, sugieren que las ocupaciones humanas del asentamiento de Santa Inés, se habrían desarrollado en asociación a un depósito arcilloso rico en diatomeas que reflejaría un lago profundo, bajo condiciones más húmedas que en el Holoceno temprano, que cambiaron hacia los 6000 años cal AP (Valero Garcés et al. 2005), y posiblemente hacia los 5700 años cal $\mathrm{AP}$, como lo sugiere el registro polínico más preciso obtenido en la cercana laguna de Aculeo (Jenny et al. 2002).

En paralelo a los estudios de polen fósil, el registro sedimentario de la ex laguna de Tagua Tagua fue evaluado para la búsqueda de carbón macroscópico. El registro muestra un marcado incremento de partículas de carbón en una posición estratigráfica equivalente a la ocupación humana más antigua de la localidad (Tagua Tagua 1), hace -13260 años cal. AP., no obstante, el pico más alto se asocia a una edad del Holoceno medio (Heusser 1983) contemporáneo a las ocupaciones aquí discutidas.

\section{Metodología}

El sitio de Santa Inés se sitúa a unos $15 \mathrm{~km}$ al SE de la ciudad de San Vicente de Tagua Tagua (Figura 1) y fue excavado en seis temporadas de terreno entre 1984 y 1987, cubriendo un área de 36 m2 (17 unidades), lo que constituyó un muestreo de gran parte del asentamiento. El sitio ha sido descrito someramente, no obstante, las únicas evidencias estudiadas corresponden a la descripción de una punta de proyectil "Cola de Pescado", sin otras asociaciones culturales (Kaltwasser et al. 1986a) y la obtención de dos fechados radiocarbónicos (Westfall et al. 2001).

Considerando la relevancia de este asentamiento y sus eventuales relaciones con otros sitio Arcaicos y alfareros de la localidad como Cuchipuy (Kaltwasser et al. 1980), Tagua Tagua II (Durán 1980, Núñez et al. 1994), Parcela 127 y el Salvador (Westfall et al. 2001), se procedió al estudio de las evidencias culturales de algunas unidades excavadas, las que incluyen instrumentos líticos, artefactos óseos, restos de fauna y evidencias bioantropológicas. 
Para contextualizar estos hallazgos se consultaron todos los cuadernos de campo de las excavaciones, lo que permitió entender la estratigrafía del sitio, las asociaciones culturales y detalles relacionados con los hallazgos. Adicionalmente, se visitó el sitio para caracterizar su emplazamiento y estado actual.

Por otra parte, se obtuvieron tres nuevas edades radiocarbónicas (AMS) sobre restos óseos de entierros Arcaicos, cuyos resultados fueron calibrados con el programa OxCal 4.01 (Bronk Ramsey 2009) usando la curva ShCal04 (McCormac et al. 2004). Sobre estas mismas muestras se realizaron análisis de isótopos estables para determinar sus valores de $\delta^{15} \mathrm{~N}$ y $\delta^{13} \mathrm{C}$.

El material lítico se estudió desde el punto de vista morfológico considerando las distintas materias primas empleadas y cadenas operativas que permitieran definir la estrategia tecnológica del conjunto lítico (Nelson 1991). Una pequeña muestra de derivados de núcleo fueron examinados bajo lupa binocular para atestiguar o no la presencia de huellas de uso (Semenov 1981). Por otra parte, el conjunto de restos faunístico, aunque se trata de una pequeña muestra, se identificó sobre la base de colecciones comparativas y se cuantificó, obteniendo los datos básicos de NISP y NMI según los criterios de Grayson (1984).

Sobre la base de los antecedentes y análisis realizados, se caracterizan las ocupaciones del sitio y se plantean algunas relaciones con aquellos asentamientos de la localidad antes mencionados.

\section{ESTRATIGRAFÍA Y CRONOLOGÍA}

El sitio arqueológico se emplaza en una terraza de la ex laguna de Tagua Tagua, a sólo unos $30 \mathrm{~m}$ de su antigua ribera. La estratigrafía muestra una primera capa de unos $30 \mathrm{~cm}$ de espesor, compuesta de arcilla y material orgánico de color pardo oscuro, donde se dispone una ocupación del período Alfarero Temprano (200 a.C. - 1000/1200 d.C.), en parte removida por la actividad antrópica actual. Una segunda capa con un espesor de $40 \mathrm{~cm}$, se compone de arcillas más finas color pardo claro, asociadas a la presencia de eventos ocupacionales de cazadores-recolectores del Holoceno Medio y Tardío. Una tercera capa, de espesor no definido, corresponde a una arcilla café amarillenta muy dura, con escasos clastos medianos y pequeños como los observados en las capas anteriores. En esta capa se registró la base de 
una punta de proyectil "Cola de Pescado", sin asociación cultural y en una situación estratigráfica y cronológica incierta (Kaltwasser et al. 1986b).

La obtención de tres nuevas fechas radiocarbónicas, junto a las dos preexistentes (Kalwasser et al. 1986b, Westfall et al. 2001), permiten precisar la cronología del asentamiento (Tabla 1 y Figura 2). La secuencia de fechas muestra para la capa II, varios eventos ocupacionales no segregados estratigráficamente correspondientes a grupos cazadores-recolectores entre los 6000 y 3700 años cal AP, mientras que la capa I atestigua, probablemente, más de un evento ocupacional del Alfarero Temprano, datado hacia los 600 años d.C.

Esta secuencia muestra un relativo sincronismo de los niveles intermedios y superiores con el cercano sitio de Cuchipuy (Kalwasser et al. 1983), así como fuertes afinidades con su contexto (Kalwasser et al. 1980, 1986), lo que sugiere ser parte de un mismo patrón de asentamiento en torno a la laguna.

\begin{tabular}{|l|l|l|l|l|l|}
\hline Código Laboratorio & $\begin{array}{l}{ }^{14} \mathbf{C} \text { años } \\
\text { AP - DC }\end{array}$ & $\boldsymbol{\delta 1 3 C ~ ( \% o ) ~}$ & $\begin{array}{l}\text { Edad calibrada } \\
\mathbf{1 \sigma}(\mathbf{a n ̃ o s} \text { cal AP) }\end{array}$ & Procedencia & Material \\
\hline *UCTL 1387 & $1380 \pm 130$ & --- & $/$ & Sondeo $(0-30 \mathrm{~cm})$ & Cerámica \\
\hline$* *$ Beta $¿ ?$ & $3720 \pm 100$ & --- & $4294(4001) 3699$ & Pozo $13(40 \mathrm{~cm})$ & Hueso Humano \\
\hline Beta 289393 & $4570 \pm 40$ & $-19,6$ & $5313(5162) 4983$ & C-5 $(40-60 \mathrm{~cm})$ & Hueso Humano \\
\hline Beta 289394 & $4820 \pm 40$ & $-19,0$ & $5644(5526) 5470$ & C-10 $(20-40 \mathrm{~cm})$ & Hueso Humano \\
\hline Beta 289396 & $5170 \pm 40$ & $-25,6$ & $5999(5930) 5758$ & P. $11(40-60 \mathrm{~cm})$ & Carbón \\
\hline
\end{tabular}

Tabla 1: edades 14C y Tl del sitio Santa Inés ( ${ }^{*}$ Westfall et al. 2001, **Kalwasser et al. 1986b).

Table 1: 14C and Tl ages for the Santa Inés site ( ${ }^{*}$ Westfall et al. 2001, **Kalwasser et al. 1986b)

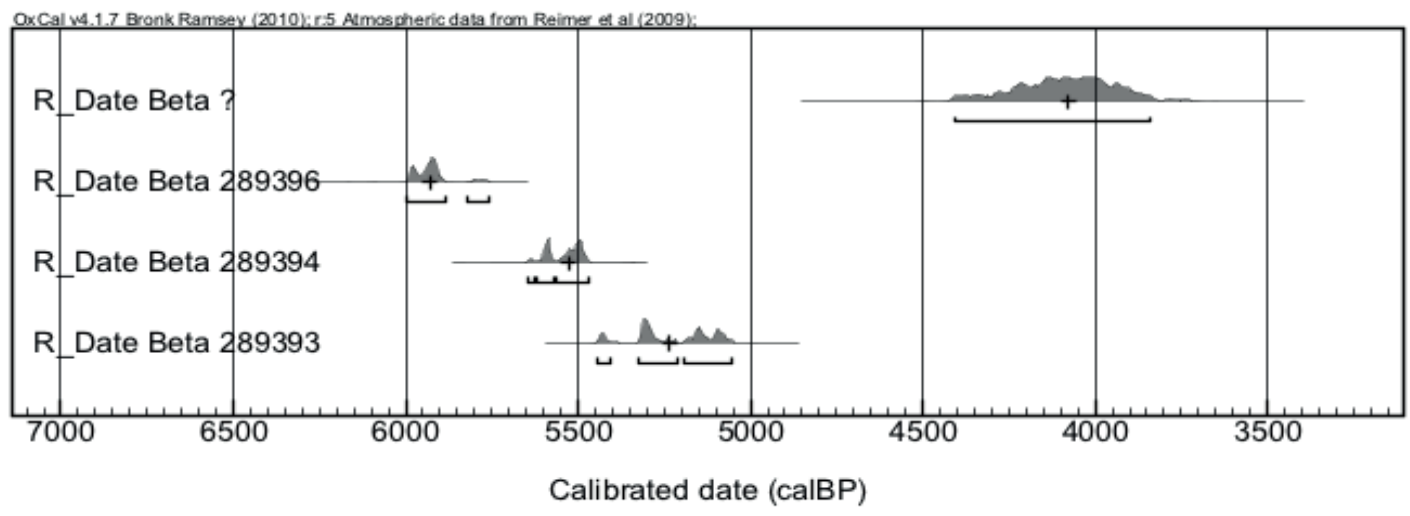

Figura 2: Rangos calibrados comparados de las edades presentadas en el trabajo.

Figure 2: Compared radiocarbon ranges of the dates presented in this work. 


\section{LAS EVIDENCIAS ARQUEOLÓGICAS Y BIOANTROPOLÓGICAS}

Para la ocupación superior del período Alfarero, el contexto muestra la presencia de cerámica representada por escasos fragmentos de cuerpos de paredes delgadas $(38 \mathrm{~mm})$, con antiplástico fino distribuido homogéneamente, superficies de color pardo a rojizo, paredes internas alisadas y externas pulidas. También se registran algunos fragmentos de "turba" cocida. Si bien en el área de estudio se conoce muy poco sobre los asentamientos alfareros, al menos se sabe que el sitio Parcela 127 y El Salvador, situados en las cercanías de Santa Inés, presentan algunas afinidades en su alfarería y cierta proximidad cronológica (Cáceres et al. 1995, Westfall et al. 2001).

Los instrumentos líticos de las ocupaciones incluyen puntas de proyectil (27 en la ocupación Arcaica y 8 en la ocupación Alfarera) apedunculadas triangulares de base cóncava, convexa y recta, además de formas lanceoladas de base convexa y cóncava elaboradas tanto sobre obsidiana, como rocas basálticas (Figura 3). Las puntas de proyectil lanceoladas de base convexa y cóncava se han registrado únicamente en los niveles arcaicos. Por otra parte, se registran también seis preformas lanceoladas elaboradas en obsidiana y basalto, así como implementos de molienda, cuchillos, lascas retocadas, algunos núcleos, cantos astillados, tajadores y piedras horadadas, éstas últimas constatadas únicamente en las ocupaciones Arcaicas.
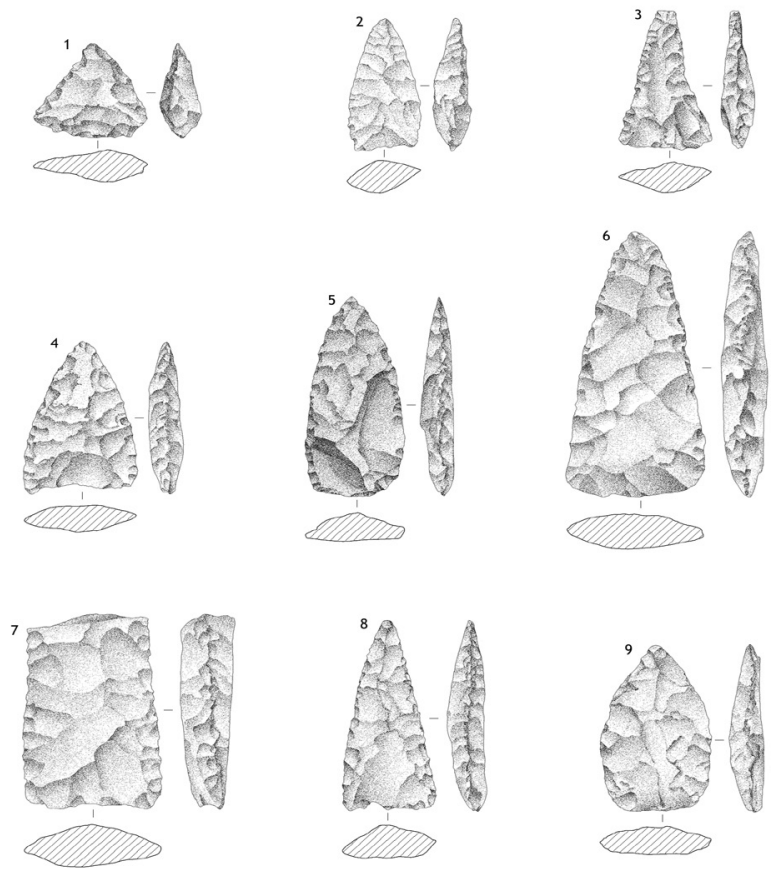

Figura 3: Puntas de Proyectiles. 1-3: Periodo Alfarero; 4-9: Periodo Arcaico. Figure 3: Projectil points. 1-3 Ceramic Period; 4-9: Archaic Period 
Los escasos derivados de núcleo registrados muestran, al menos, que a lo largo de todas las ocupaciones se procesaron algunos guijarros de materias primas locales (basaltos), cuyas lascas ocasionalmente fueron utilizadas como instrumentos de filos vivos. Esto se corrobora a través de la presencia de micro-huellas de uso (microastillamiento y desgaste) en los mismos instrumentos. La presencia de preformas bifaciales (Figura 4) y desechos de retoque sugieren el procesamiento local de algunos cabezales líticos (basalto) y el reactivado de filos desgastados (desechos de obsidiana y sílice).

La frecuencia absoluta de materias primas en desechos (Figura 5) muestra una preponderancia sobre obsidiana, en contraste con la frecuencia de materias primas en puntas de proyectiles (Figura 6), donde predomina el sílex, basalto y otras rocas. Esto sugiere que la mayor frecuencia de desechos pequeños de obsidiana son el resultado esencialmente del reactivado de puntas de proyectiles que llegaron al sitio, ya sea como preformas o piezas terminadas.

Las características de las evidencias líticas, tanto para las ocupaciones Arcaicas, como Alfareras, sugieren una tecnología esencialmente curatorial (Nelson 1991), tanto en las materias primas locales, como no locales, no obstante, la presencia de instrumentos de filos vivos en materias primas locales indican una estrategia expeditiva para tareas genéricas de escasa planificación.

Entre los instrumentos de hueso, para la ocupación Alfarera se registraron cinco fragmentos de probables leznas (Figura 4). Cuatro de éstos corresponden a la sección del "cuerpo" y uno al extremo distal. Por otra parte, para los eventos ocupacionales Arcaicos, se constató un total de 9 fragmentos de cuerpos y extremos distales de instrumentos de hueso, probablemente también correspondientes a leznas con superficies pulidas y varios de ellos con huellas de exposición al fuego, así como un fragmento de hueso largo (camélido) con huellas de cortes y desgaste (¿retocador?). Al menos tres de estas piezas se registraron bajo un entierro. 

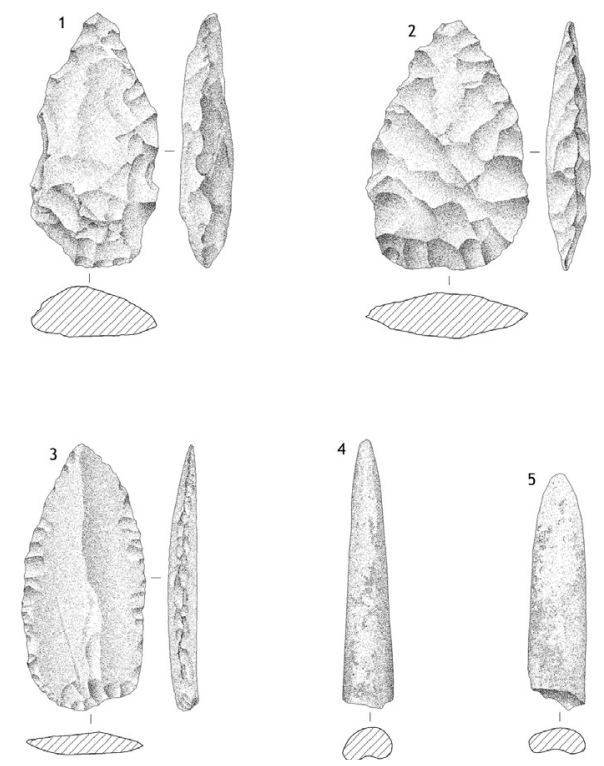

Figura 4. Instrumentos Liticos. Periodo Alfarero: 1) Preforma, 2) Cuchillo, 4-5) Leznas de Hueso. Período Arcaico: 3) Cuchillo.

Figure 4: Lithic instruments. Ceramic Period: 1) preform, 2) knife, 4-5) Bone awls. Archaic Period: 3) Knife.

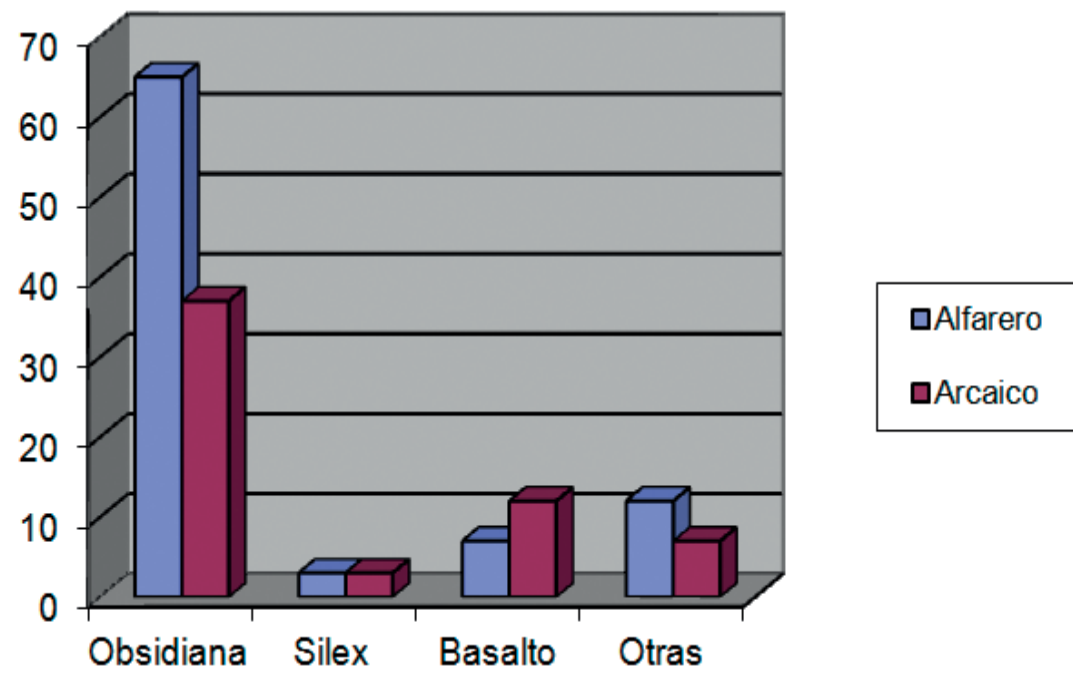

Figura 5: Frecuencia de materias primas en desechos liticos.

Figure 5: Raw Material frequency of lithic debitage.

Por otra parte, los restos óseos recuperados en todos los niveles de la cuadrícula 10 y de los pozos 11 y 12, permitieron el análisis taxonómico y cuantitativo de las evidencias de restos faunísticos. La determinación 
taxonómica incluyó la presencia de rana (Calyptocephalella gayi), coipo (Myocastor coipus), roedores (Octodon sp.), peces (Percichthys trucha) y una especie de ave no identificada (Tabla 2). Al parecer todos los restos se incorporaron antrópicamente al contexto y muestran fractura y astillamiento intencional, así como indicios de exposición al fuego con excepción de los restos de Octodon sp. Tanto para la ocupación Alfarera, como Arcaica las especies predominantes (NMI) fueron Myocastor coipus y Calyptocephalella gayi. Únicamente Percichthys trucha y Octodon sp, están ausentes en la ocupación Arcaica. Así también, se constató en los niveles ocupacionales Arcaicos, la presencia de algunos fragmentos de molusco correspondientes a un bivalvo (Protothaca thaca) de origen marino. Adicionalmente, en otras cuadrículas no cuantificadas, se observó la presencia de guanaco (Lama guanicoe) representado por escasos fragmentos de huesos largos.

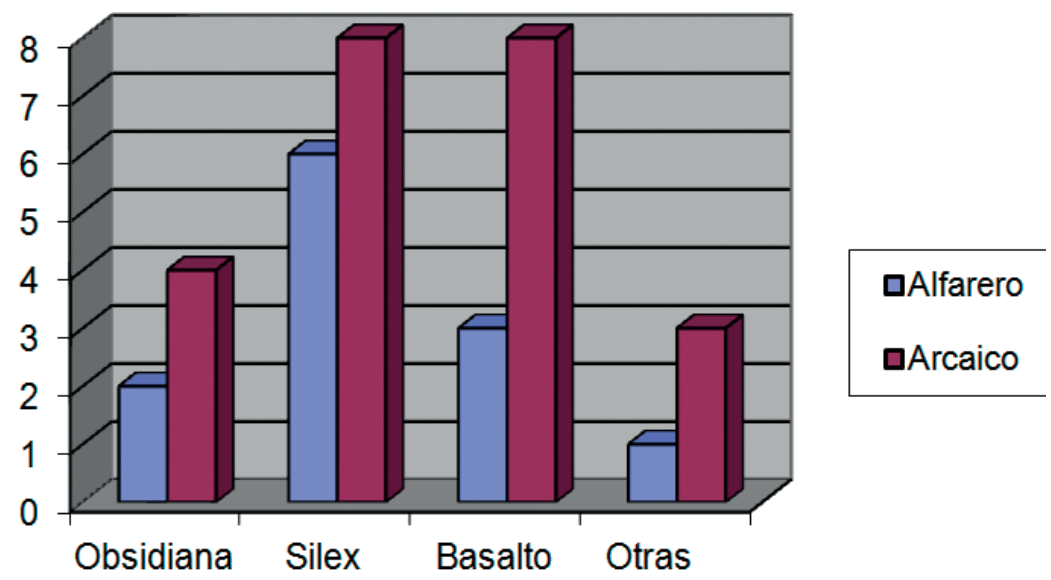

Figura 6: Frecuencia de materias primas en puntas de proyectiles

Figure 6: Raw material frequency for projectile points.

\begin{tabular}{|c|c|c|c|}
\hline Capas & Identificación Taxonómica & NISP & NMI \\
\hline Alfarero & Calyptocephalella gayi & 26 & 6 \\
\hline & Myocastor coipus & 38 & 6 \\
\hline & Percichthys trucha & 3 & 3 \\
\hline & Octodon sp. & 3 & 2 \\
\hline Arcaico & Ave no identificada & 16 & 3 \\
\hline & Caudiverbera caudiverbera & 11 & 3 \\
\hline & Myocastor coipus & 17 & 4 \\
\hline & Ave no identificada & 6 & 1 \\
\hline
\end{tabular}

Tabla 2: Restos de fauna en unidades 11, 12 y cuadricula 10.

Table 2: Faunal remains from Units 11.12 and Square 10. 
Estas exiguas evidencias sugieren, al menos, desde el punto de vista cinegético, que existieron similares patrones de consumo en los dos componentes ocupacionales. Probablemente se desarrolló una estrategia que privilegió la obtención de recursos acuáticos de mayor biomasa y contenido proteico (Myocastor coipus), complementándolos con aquellos más pequeños, pero abundantes y de fácil obtención (Calyptocephalella gayi).

Por otra parte, las excavaciones del sitio permitieron recuperar un total de 17 entierros, la gran mayoría de estos se encontraban flexionados y ocasionalmente sobrepuestos. Éstos se sitúan entre los 78 y $40 \mathrm{~cm}$ de profundidad. En algunos casos, los individuos fueron cubiertos con guijarros y clastos angulosos a modo de pequeños túmulos, como se observa en los niveles intermedios de Cuchipuy (Kalwasser et al. 1980). Sólo excepcionalmente se registraron ofrendas, constituidas por puntas de proyectil, algunas con pigmento rojo; piedras horadadas, tajadores, cuchillos, lascas de obsidiana y algunos instrumentos de hueso. Sólo uno de estos entierros podría corresponder eventualmente al período Alfarero, mientras que los restantes, por su ubicación estratigráfica, son atribuidos a los períodos Arcaico Medio y Tardío.

Las fechas directas de tres esqueletos muestran que se ubican cronológicamente hacia el Arcaico Medio (5999 y 4983 años cal AP.) y Arcaico Tardío (4294 - 3699 años cal AP.) (Tabla 1). Por su parte, los valores de los análisis de isótopos estables de carbono $\left(\delta^{13} \mathrm{C}\right)$ y nitrógeno $\left(\delta^{15} \mathrm{~N}\right)$ de dos muestras (Tabla 3 ) evidencian un consumo principalmente de dieta terrestre, con ingesta de proteína animal y plantas del tipo C3 para los dos individuos analizados. Con respecto a las señales de nitrógeno de ambos esqueletos se observa que caen dentro del rango esperado para un consumo de dieta terrestre (Barberena 2002), muy similar a valores obtenidos para otros grupos de cazadores recolectores de la zona de Chile Central y en consistencia con la información de ecología isotópica actualmente disponible para la región (Falabella et al. 2007).

Al comparar las señales isotópicas de ambos individuos, se observa que la señal de nitrógeno del esqueleto 2 proveniente del nivel $40-60 \mathrm{~cm}$, se encuentra empobrecida con respecto a la del Esqueleto 1 (Tabla 3). Esta situación podría deberse a un mayor consumo de plantas C3 que de proteína animal por parte del primer individuo, o bien, a un consumo de fauna terrestre de bajo nivel trófico. A diferencia de lo que ocurre con el esqueleto del nivel 40-60, el Esqueleto 1 presenta valores más enriquecidos 
de nitrógeno, lo que podría ser explicado por el consumo de fauna terrestre de nivel trófico más alto o porque alguna de las especies consumidas, pero no documentadas en el contexto arqueológico, posea una dieta omnívora.

Las señales isotópicas se correlacionan con la evidencia obtenida a partir del registro arqueológico del sitio, en donde se han encontrado principalmente restos de fauna terrestre, tales como aves, roedores y guanaco, y también restos de fauna lagunar tales como batracios, coipo, y algunos restos de peces.

Cabe destacar que los valores isotópicos de nitrógeno y carbono para fauna de ambientes lagunares pueden evidenciar enriquecimiento en sus señales, debido a la presencia de cadenas tróficas más largas dentro de las lagunas que aquellas terrestres (Katzenberg y Weber 1999). Sin embargo, esta situación no ha sido estudiada en detalle para el caso de las lagunas en Chile Central.

\begin{tabular}{|l|c|c|}
\hline \multicolumn{1}{|c|}{ Muestra } & ${ }^{\delta 13} \mathbf{C}$ & ${ }^{15} \mathbf{N}$ \\
\hline Unidad C-5 (Esqueleto 2) nivel 40-60 cm. & $-19,6 \% 0$ & $6,8 \% 0$ \\
\hline Unidad C-10 (Esqueleto 1) nivel 20-40 cm. & $-19,0 \% 0$ & $9,6 \% 0$ \\
\hline
\end{tabular}

Tabla 3. Valores isotópicos de carbono y nitrógeno en esqueletos humanos.

Table 3: Carbon and Nitrogen Isotopic values in human remains.

\section{DisCUSIÓN Y CONCLUSIONES}

El sitio de Santa Inés constituye un relevante asentamiento en consideración de su redundancia ocupacional y contextos funerarios, así como por sus eventuales relaciones con otros asentamientos cercanos emplazados en torno a la laguna de Tagua Tagua. Este emplazamiento sin dudas constituyó un ambiente circunscrito de recursos estables que fue privilegiado para el asentamiento a lo largo de gran parte del Holoceno.

En la historia ocupacional de este asentamiento, las primeras evidencias detectadas corresponden a un fragmento basal de punta de proyectil "Cola de Pescado" de factura claramente Paleoindia, sin embargo, no presenta asociaciones culturales y su situación estratigráfica y cronológica es incierta (Kaltwasser et al. 1986b). Este hallazgo detectado en la capa inferior del asentamiento, podría ser explicado como pérdida de un cabezal lítico durante las actividades cinegéticas en torno a la laguna por grupos cuyas evidencias han sido fechadas hacia los 13233 - 12693 y 11803 - 11233 años cal AP en 
los sitios próximos de Tagua Tagua 1 y 2, respectivamente (Montané 1968, Núñez et al. 1987, 1994; Méndez 2010).

Con posterioridad, hacia los 5999 y 4983 años cal AP., cuando imperaban condiciones climáticas más húmedas que en el Holoceno temprano y un lago profundo, el asentamiento de Santa Inés es ocupado por primera vez en forma consistente, como campamento habitacional con varios eventos ocupacionales asociados a prácticas funerarias.

Las evidencias culturales muestran a grupos cazadores recolectores con puntas de proyectil triangulares y lanceoladas elaboradas en materias primas locales y extra-locales (obsidiana, sílex y cristal de cuarzo), asociados a frecuentes entierros humanos. Las prácticas cinegéticas atestiguan la caza de mamíferos terrestres (Lama guanicoe) y el aprovechamiento de recursos lacustres, con especial énfasis en presas de mayor biomasa y contenido graso (Myocastor coipus), además de ranas, aves y algunos peces. Al parecer, en estos momentos habrían ocurrido movimientos hacia la costa, como lo evidencian algunos restos de moluscos, sin embargo, la dependencia de recursos marinos no habría sido sustantiva.

En un segundo momento ocupacional datado hacia los 4294 - 3699 años cal AP., cuando las condiciones climáticas se hacen cada vez más favorables, el asentamiento sigue siendo ocupado como un campamento habitacional por grupos cazadores recolectores, esta vez, portadores únicamente de puntas de proyectiles triangulares apedunculadas. Existen escasas evidencias de entierros y tampoco se encuentran indicadores de contactos con la costa.

Ambas ocupaciones Arcaicas son correlacionables cronológica y culturalmente con los niveles intermedios de Cuchipuy datados hacia los 8870 a 5750 años cal AP. y probablemente también con los niveles tardíos de este mismo sitio, el cual se localiza a sólo $5,5 \mathrm{~km}$ al Este de Santa Inés. Con este cercano sitio comparten fuertes afinidades culturales y contextuales. No obstante, las puntas triangulares de Santa Inés tienden a ser de menor tamaño. Estas características tipológicas y tecnológicas, hacen suponer una misma unidad cultural.

En este marco, tanto Santa Inés como Cuchipuy, constituyen sitios habitacionales con contextos funerarios reiterados que muestran que estos asentamientos también fueron utilizados como cementerio. Este tipo de yacimientos podría estar reforzando la suposición de unidades sociales distintas, donde los espacios rituales para entierro, marcan y distinguen 
identidades de bandas unidas por lazos de parentesco, apropiando y diferenciando dicho espacio. Destaca el hecho de que en esta locación de recursos privilegiados se den los primeros indicios regionales de reiteración en el asentamiento y entierro entre grupos de cazadores recolectores. Esto permitiría suponer una programación de "dónde" llevar a cabo al menos algunos de los entierros, lo que es indicador de un proceso inicial de restricción de la movilidad.

Con posterioridad a las últimas ocupaciones arcaicas, y con un hiato cronológico de un par de milenios, se reocupa el asentamiento hacia los $1380 \pm 130$ años A.P. (Westfall et al. 2001) por grupos del período Alfarero Temprano. El sitio es ocupado como un asentamiento habitacional a juzgar por la variabilidad artefactual, así como por la presencia de "turba" cocida, que sugiere la presencia de estructuras algo más permanentes. Se mantiene una tecnología lítica curatorial y ósea similar a las ocupaciones anteriores, la que es también consistente con una similar fauna explotada. Sólo excepcionalmente ocurren prácticas funerarias. Por otra parte, contextos contemporáneos, cercanos a Santa Inés como Parcela 127, El Salvador y Cuchipuy (Cáceres et al. 1995, Westfall et al. 2001), sugieren que la laguna de Tagua Tagua continuó siendo un polo de atracción para la instalación de asentamientos humanos durante dicho período.

Las evidencias de las ocupaciones Arcaicas de Santa Inés y Cuchipuy, así como Tagua Tagua II (Durán 1980), junto con aquellas ya mencionadas del periodo Alfarero Temprano, muestran, desde el Holoceno Temprano al Tardío, una reiterada ocupación humana en torno a la ex-laguna de Tagua Tagua. Estas ocupaciones se hacen aparentemente más intensas y reiteradas durante el Holoceno Medio y Tardío, cuando las condiciones climáticas regionales indican un incremento de la humedad, constituyendo en esos momentos un hábitat con recursos predecibles y abundantes. Esto hace que la cuenca se visualice como un polo de atracción, primero para los grupos cazadores-recolectores y, posteriormente, para los grupos Alfareros de Chile Central, generando un patrón de asentamiento reiterado en torno a la laguna, el que puede ser caracterizado como una adaptación circunlacustre.

Agradecimientos: Esta investigación forma parte del proyecto A/023587/09 del Programa de Cooperación Interuniversitaria e Investigación Científica (Secretaría de Cooperación Internacional, España) entre la Universitat Rovira i Virgili (Tarragona) y la Universidad de Chile. 


\section{BibliografíA}

Barberena, R. 2002. Los Limites del Mar: Isótopos Estables en Patagonia Meridional. Colección Tesis de Licenciatura. Sociedad Argentina de Antropología. Buenos Aires.

Bronk Ramsey, C. 2009. "Bayesian analysis of radiocarbon dates". Radiocarbon 51: 337-360.

Cáceres I.; F. Gallardo y P. Miranda. 1995. "Prehistoria, Asentamiento y Paleoecología en la cuenca del Río Cachapoal, Chile Central: Un balance regional”. Gaceta Arqueológica Andina 24:173-193.

Cáceres I.; C. Westfall y F. Gallardo. 2000. "Asentamientos Cerámicos Tardíos en el curso medio del río Cachapoal, Chile Central”. Actas del $2^{\circ}$ Taller de Arqueología de Chile Central. Santiago, Chile. (Disponible en http://www. geocities.com/actas2taller/ivan2.htm.)

Darwin, C. [1909] 2004. The Voyage of the Beagle. Barnes and Noble, New York.

Durán, E. 1980. “Tagua Tagua II, Nivel de 6.130 años: Descripción y Relaciones”. Boletín Museo Nacional de Historia Natural, 37: 75-86.

Falabella F.; M. Planella, E. Aspillaga, L. Sanhueza y R. Tykot. 2007. “Dieta en sociedades Alfareras de Chile Central: Aporte de análisis de isótopos estables". Chungara, Revista de Antropología Chilena 39 (1): 5-27.

Fuenzalida V. 1965. Biogeografía. Geografía Económica de Chile. Capítulo VII, Corfo, Santiago.

Gay, C. [1831] 1911. "Memoria de 20 de marzo de 1831 dirigida a la Comisión Científica Chilena". En Don Claudio Gay; su vida y sus obras. Centro Diego Barros Arana, Santiago.

Grayson, D. 1984. Quantitative zooarchaeology. Topics in the Analysis of Archaeological Faunas. Academic Press, New York.

Heusser, C. 1983. "Quaternary pollen record from Laguna de Tagua Tagua, Chile”. Science 219: 1429-1431.

----- 1990. "Ice age vegetation and climate of subtropical Chile". Palaeogeography, Palaeoclimatology, Palaeoecology 80:107-127.

Jenny, B.; B. Valero-Garcés, R. Villa-Martínez, R. Urrutia, M. Geyh y H. Viet. 2002. "Early to mid-Holocene aridity in Central Chile and the southern westerlies: The Laguna Aculeo record (340S)". Quaternary Research 58: $160-170$. 
Katzenberg, A. y Weber, E. 1999. "Stable Isotope Ecology and Paleodiet in the Lake Baikal, Region of Siberia”. Journal of Archaeological Science 26: 651659 .

Kalwasser, J.; A. Medina y J. Munizaga. 1980. "Cementerio del Período Arcaico en Cuchipuy”. Revista Chilena de Antropología 3:109-123.

1983. "Estudio de once fechas de R.C. 14 relacionadas con el hombre de Cuchipuy". Boletín de Prehistoria de Chile 9: 9-13.

Kalwasser, J.; A. Medina, E. Aspillaga y C. Paredes. 1986a. "El Hombre de Cuchipuy: Prehistoria de Chile Central en el Período Arcaico". Chungara, Revista de Antropología Chilena 16-17:99-105.

Kalwasser J.; A. Medina, E. Aspillaga e I. Cáceres. 1986b. "Punta Cola de Pescado encontrada en Chile Central". Revista Chilena de Antropología 5:1116.

Luebert, F. y P. Pliscoff. 2006. Sinopsis Bioclimática y Vegetacional de Chile. Editorial Universitaria, Santiago.

McCormac, F.G.; Hogg, A.G.; Blackwell, P.G.; Buck, C.E.; Higham, T.F.G.; Reimer, P.J. 2004. "SHCal04 Southern Hemisphere calibration, 0-11.0 cal kyr BP”. Radiocarbon 46(3): 1087-1092.

Méndez, C. 2010. Tecnología Litica en el Poblamiento Pleistoceno Terminal del Centro de Chile, Organización, Gestos y Saberes. Tesis Doctoral inédita, Universidad Católica del Norte - Universidad de Tarapacá, San Pedro de Atacama.

Montané, J. 1968. "Paleo-indian remains from Laguna Taguatagua, Central Chile". Science 161: 1137-1138.

Nelson, M. 1991. "The study of technological organization”. En Archaeological Method and Theory, editado por M. Schiffer, vol 3: 57-100. University of Arizona Press, Tucson.

Núńez L.; J. Varela y R. Casamiquela. 1987. "Ocupación Paleoindia en el Centro-Norte de Chile: Adaptación circunlacustre en las tierras bajas". Estudios Atacameños 8: 142-185.

Núñez, L.; J. Varela, R. Casamiquela, V. Schiappacasse, H. Niemeyer y C. Villagrán. 1994. "Cuenca de Taguatagua en Chile: El Ambiente del Pleistoceno Superior y Ocupaciones Humanas". Revista Chilena de Historia Natural 67:503-519.

Ovalle, A. de [1646]1969. Histórica Relación del Reyno de Chile. Instituto de Literatura Chilena, Santiago. 
Rosales, D. de [1670] 1877. Historia General del Reyno de Chile. Tomo I. Imprenta i Librería del Mercurio, Valparaíso.

Semenov, S. A. 1981. Tecnología Prehistórica. Editorial Akal, Madrid.

Valero-Garcés, B.; B. Jenny, M. Rondanelli, A. Delgado-Huertas, S. Burns, H. Veit y A. Moreno. 2005. "Palaeohydrology of Laguna de Tagua Tagua $\left(34^{\circ} 30^{\prime} \mathrm{S}\right)$ and moisture fluctuations in Central Chile for the last 46.000 yr". Journal of Quaternary Science 20(7-8): 625-641.

Varela, J. 1976a. "Geología del Cuaternario de la Laguna de Taguatagua (Prov. de O’Higgins)”. Actas del I Congreso Geológico, pp: 81-112. Santiago, Chile.

----- 1976b. Estudio estratigráfico-sedimentológico de los depósitos de Laguna Taguatagua. Provincia de O'Higgins. Tesis de prueba para optar al título de geólogo, Universidad de Chile, Santiago.

Westfall C.; I. Cáceres y A. Román. 2001. "Nuevos fechados del Período Cerámico en la Ex-Laguna de Tagua Tagua, Chile Central". Werken 2: 3746. 